\title{
Families of Distributions for Hourly Median Power and Instantaneous Power of Received Radio Signals
}

\author{
M. M. Siddiqui ${ }^{1}$ and George H. Weiss
}

(Received June 13, 1963)

\begin{abstract}
In this paper the gamma family of probability distributions is studied in connection with the distribution of hourly median received power or transmission loss. By taking a mixture of Rayleigh distributions with gamma distributions as mixing distributions the long-term distributions of instantaneous signal power are theoretically derived. These distributions are evaluated in closed form under several hypotheses. The question of estimating the parameters is discussed. Graphs and tables are prepared to facilitate the application of the theory to the data.
\end{abstract}

\section{Introduction}

When incoherent scatter is the dominant mode of radio wave propagation, as is the case with VHF and UHF tropospheric transmission, the distribution functions of samples of instantaneous or hourly median received power show a great deal of variability. Some of the variability, ascribable to some obvious physical parameters such as the diurnal and seasonal variation and the angle of transmission, may be separated out from the data. However, due to the sheer complexity of the physical mechanism involved, most of the variability in the data could only be studied statistically. Since on certain statistical hypotheses the sum of a great many random variables is normally distributed, it has been assumed that the short-term distribution of instantaneous received power, $z$, is Rayleigh distributed. In statistical literature the Rayleigh distribution is more familiar under the name of exponential distribution or $\chi^{2}$ distribution with $2 \mathrm{deg}$ freedom. The Rayleigh distribution, however, does give a good fit neither to the long-term distribution of instantaneous power, $z$, nor to the distribution of short-term (say hourly) mean or median power, $q$, of the received signal. In previous work on this subject it has been tentatively assumed that $q$ is lognormally distributed.

In this paper we consider an alternate family of distributions to lognormal, i.e., the two parameter gamma family. The arguments in favor of choosing' this particular family are the following. First, among the various distributions which could be defined on the positive real line $(0, \infty)$ the gamma family is a natural extension of the Rayleigh distribution. Second, the generalized Laguerre polynomials associated with the function $e^{-x} x^{\alpha}$ form a complete orthogonal basis for the space of functions which are integrable over $(0, \infty)$. Hence, choosing an appropriate gamma probability density function will give a zeroth order approximation to the probability density function of any positive random variable, such as the power or amplitude of a vector.

\footnotetext{
1 Boulder Laboratories, National Bureau of Standards, Boulder, Colo.
}

If further approximations are desired, they could be easily obtained in terms of partial sums of the Laguerre polynomial expansion. Third, if we assume that $x=\lambda q^{\beta}, \lambda>0, \beta \neq 0$, is a gamma variate, we obtain a family of distributions which has great flexibility to meet the demands of the variously shaped empirical distributions of $q$. Fourth, the assumption that $q$ or $1 / q$ is a gamma variate leads to the distribution of $z$ which can be evaluated in closed form, whereas the lognormal assumption for $q$ (hence for $1 / q$ as well) leads to an integral which remains intractable. Lastly, of course, the final criterion between several hypotheses is a "goodness of fit" test to the actual data. A comparison of the general shapes of the theoretical distributions with those of the empirical distributions suggests that in the majority of cases the gamma family may give a better fit to the data than the lognormal. However, the lognormal family cannot be completely ruled out. Thus, at present, it seems desirable to keep the statistical approach flexible enough to include both these families in the analysis of scatter propagation data.

\section{Distribution of Hourly Median Received Power}

In NBS Technical Note Number 43 entitled, "A survey of VHF and UHF tropospheric transmission loss data and their long-term variability," Williamson et al. [1960] present empirical distributions of hourly median basic transmission loss for 135 beyondline-of-sight radio paths in the United States. Transmission loss is defined as the ratio of total radiated power to resulting signal power available from the receiver. All data are reported in terms of basic transmission loss, defined as the transmission loss expected between isotropic antennas, and expressed in decibels. The empirical distribution function is plotted on the normal probability paper as it is tentatively assumed that the hourly median transmission loss will be lognormally distributed. A survey of the graphs indicates, however, that most of them are not straight lines. Broadly speaking, the 


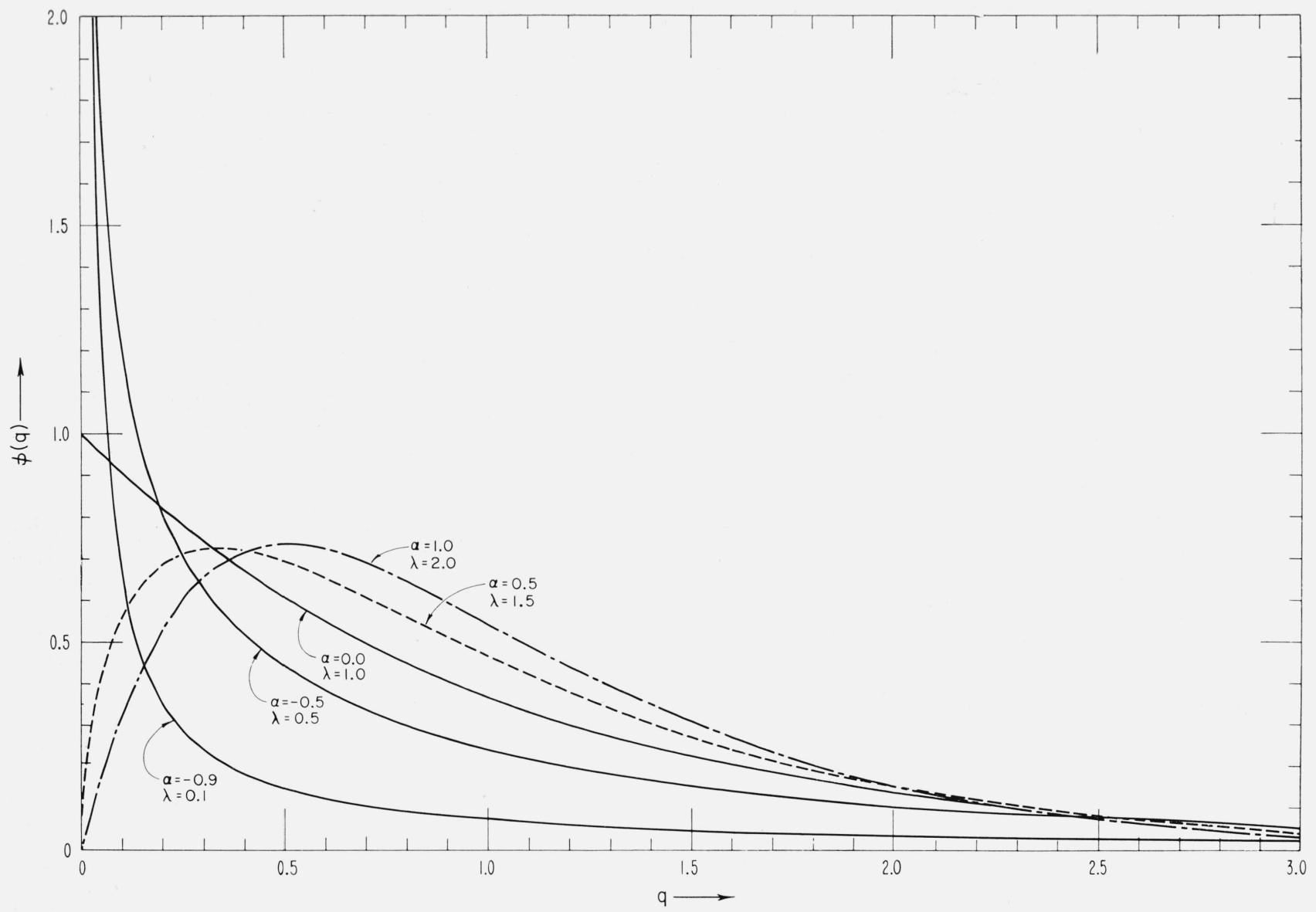

FIGURE 1. $\phi(\mathrm{x})$ versus $\mathrm{x}$ for selected values of $\lambda$ and $\alpha$.

In each case mean of $x=1$.

graphs tend to fall in the following three categories:

Category 1. Straight lines.

Category 2. Convex curves.

Category 3. Concave curves.

Again, generally speaking, it seems that convex curves are more numerous than straight lines or concave curves, although the proportion of the latter mentioned curves is significant. A very few exceptional curves have inflexion points, and, for the present, we will ignore them. To give more precise meanings to this discussion, we introduce the following notation:

$$
\begin{aligned}
q & =\text { hourly median received power, } \\
q_{0} & =\text { long-term median of } q, \\
Q & =10 \log _{10}\left(q / q_{0}\right), \\
G(y) & =\operatorname{Pr}(q \geq y), G_{1}(y)=\operatorname{Pr}(Q \geq y) .
\end{aligned}
$$

Now if $Q$ is normally distributed so is $\beta Q+\gamma$, where $\beta \neq 0$ and $\gamma$ are arbitrary real numbers. This is to say that if $q$ is lognormally distributed so is $\lambda q^{\beta}$, where $\lambda>0$, and $\beta \neq 0$ are arbitrary. In particular, since $1 / q$ is proportional to the transmission loss, setting $\beta=-1$, we have that the hourly median transmission loss is lognormally distributed if $q$ is.
Let

$$
p=1 / q, P=-Q, G_{2}(y)=\operatorname{Pr}(P \geq y),
$$

then

$$
G_{2}(y)=\operatorname{Pr}(Q \leq-y)=1-G_{1}(-y) .
$$

Thus if the graph of $G_{1}(y)$ on the normal probability paper is a straight line so is the graph of $G_{2}(y)$. On the other hand, if the graph of $G_{1}(y)$ on this paper is a convex curve the graph of $G_{2}(y)$ will be concave and vice versa.

Let $x=\lambda q^{\beta}, \lambda>0, \beta \neq 0$. In this paper we propose the family of gamma distributions specified, for each $\alpha>-1$, by the probability density function

$$
\phi_{1}(x)=\frac{x^{\alpha} e^{-x}}{\Gamma(\alpha+1)}, \quad 0<x<\infty,
$$

for the random variable $x$. In figures 2,3 , and 4

$$
\Phi_{1}(x)=\int_{x}^{\infty} \phi_{1}(t) d t
$$

is plotted on the normal probability paper against $y=10 \beta\left(\log _{10} q-\log _{10} q_{0}\right)$, where $q_{0}$ is the median of the 


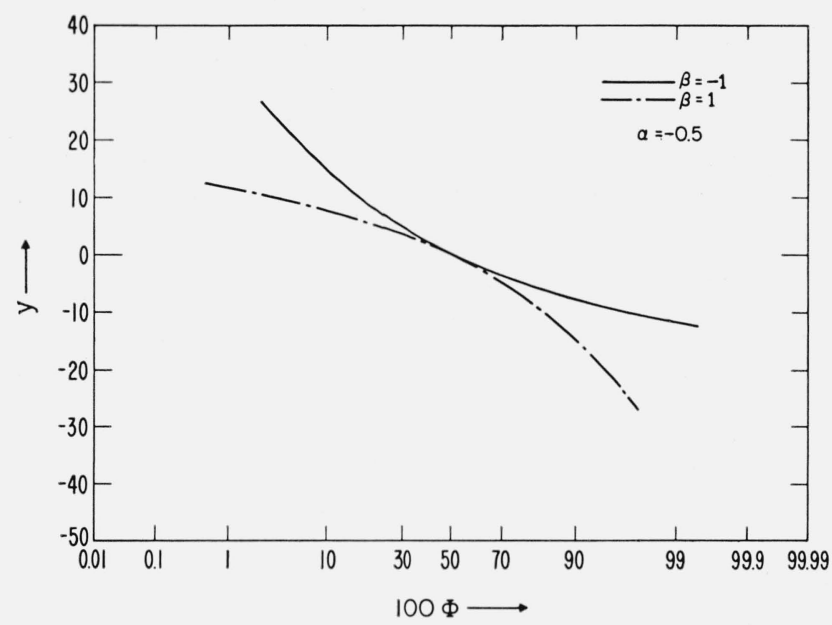

Figure 2. $100 \Phi(\mathrm{x})$ versus $\mathrm{y}=10 \beta\left(\log \mathrm{q}-\log \mathrm{q}_{0}\right)$ for $\alpha=-0.5$, where $\mathrm{x}=\lambda \mathrm{q}^{\beta}$, and $\mathrm{q}_{0}=$ median of $\mathrm{q}$.

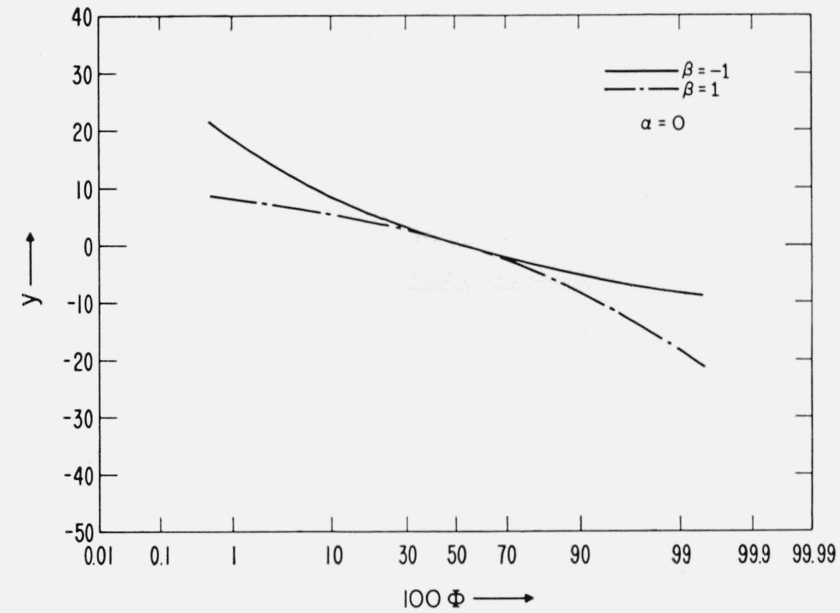

Figure 3. $100 \Phi(\mathrm{x})$ versus $\mathrm{y}=10 \beta\left(\log \mathrm{q}-\log \mathrm{q}_{0}\right)$ for $\alpha=0$, where $\mathrm{x}=\lambda \mathrm{q}^{\beta}$ and $\mathrm{q}_{0}=$ median of $\mathrm{q}$.

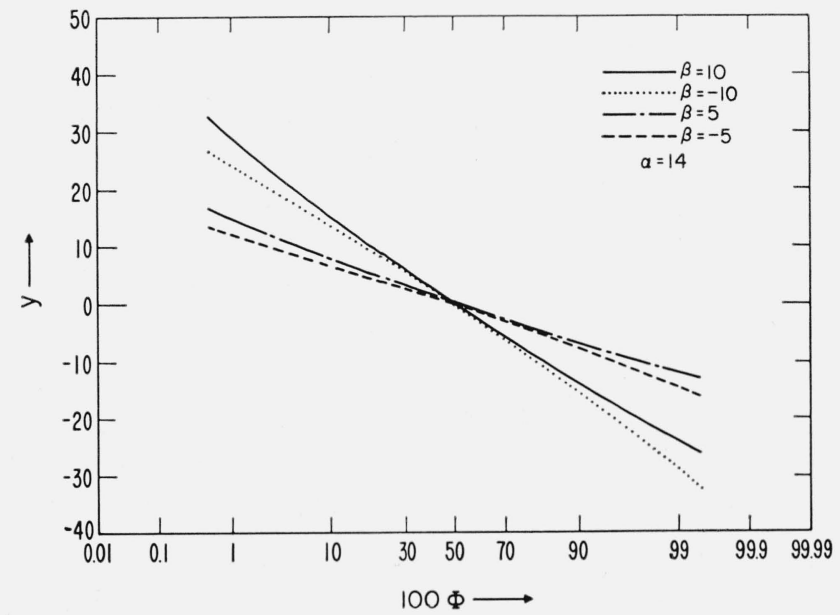

Figure 4. $100 \Phi(\mathrm{x})$ versus $\mathrm{y}=10 \beta\left(\log \mathrm{q}-\log \mathrm{q}_{0}\right)$ for $\alpha=14$, where $\mathrm{x}=\lambda \mathrm{q}^{\beta}$ and $\mathrm{q}_{0}=$ median of $\mathrm{q}$. distribution of $q$, for selected values of $\alpha$ and $\beta$. From the shapes of these graphs it is reasonable to conclude that distributions chosen from this family would give satisfactory fits to the curves of categories 2 and 3 .

\section{Estimation of Parameters}

In this paper we will confine our attention only to the case when $\beta=1$ or -1 . We will therefore assume that either $q$ or $p$ is a gamma variate with probability density function

$\phi(x)=\frac{\lambda^{\alpha+1}}{\Gamma(\alpha+1)} x^{\alpha} e^{-\lambda x}, \quad \lambda>0, \alpha>-1,0<x<\infty$.

Note the change in notation for $\phi(x)$. Now we have included $\lambda$ explicitly in the expression for $\phi(x)$. In figure $1, \phi(x)$ is plotted on arithmetic scale for selected values of $\alpha$ and $\lambda$.

Let $x_{1}, \ldots, x_{N}$ be a random sample from $\phi(x)$. The question of how to obtain such a sample in practice where, in general, successive observations are correlated is deferred to section 5. To solve the mathematical problem of estimation we may proceed in several ways. The most efficient method, as fully discussed by Fisher [1922] and recently studied by Greenwood and Durand [1960], is the method of maximum likelihood. We will outline this method briefly. Let $A$ be the arithmetic mean and $G$ the geometric mean of $x_{1}, \ldots, x_{N}$, i.e.,

$$
A=N^{-1} \Sigma x_{i}, G=\left(\amalg x_{i}\right)^{1 / N} .
$$

The natural logarithm, $L$, of the likelihood function (the joint probability density of $x_{1}, \ldots, x_{N}$ considered as a function of the parameters $\alpha$ and $\lambda$ ) is given by

$$
\begin{gathered}
L(\alpha, \lambda)=\Sigma \ln \phi\left(x_{i}\right)=N[(\alpha+1) \ln \lambda-\ln \Gamma(\alpha+1) \\
+\alpha \ln G-\lambda A],
\end{gathered}
$$

which shows that $(A, G)$ are jointly sufficient for $(\alpha, \lambda)$. Differentiating (3.3) with respect to $\alpha$ and $\lambda$ and equating the resulting expressions to zero, we must have $\hat{\alpha}$ and $\hat{\lambda}$ as the solutions of the equations

$$
(\alpha+1) / \lambda=A, \psi(\alpha+1)-\ln \lambda=\ln G,
$$

where $\psi(x)=\Gamma^{\prime}(x) / \Gamma(x)$ is a tabulated function [Davis 1933]. Taking the logarithm of the first equation and subtracting the second, we obtain the pair of equations

$$
\lambda=(\alpha+1) / A, \ln (\alpha+1)-\psi(\alpha+1)=\ln (A / G) .
$$

In table 1 we present an abstract of table 1a from Greenwood and Durand.

To use this table, write $\rho=\alpha+1, \eta=\ln \rho-\psi(\rho)$, and $y=\ln A-\ln G$. We then see that (3.4) can be written

$$
\begin{aligned}
& \eta=y \\
& \lambda=\frac{\rho}{4} .
\end{aligned}
$$




\begin{tabular}{|c|c|c|c|c|c|c|c|}
\hline$\eta$ & $\eta \rho$ & $\eta$ & $\eta \rho$ & $\eta$ & $\eta p$ & $\eta$ & $\eta p$ \\
\hline .00 & .50000 & & & & & & \\
\hline 01 & 166 & .36 & .55235 & .71 & .59049 & 1.06 & .62019 \\
\hline 02 & 331 & .37 & 359 & .72 & 144 & 1.07 & 094 \\
\hline .03 & 495 & .38 & 482 & .73 & 238 & 1.08 & 169 \\
\hline 04 & 658 & .39 & 605 & .74 & 332 & 1.09 & 244 \\
\hline 05 & 819 & .40 & 726 & .75 & 425 & 1.10 & 318 \\
\hline 06 & 979 & .41 & 846 & .76 & 517 & 1.11 & 392 \\
\hline 07 & .51139 & .42 & 965 & .77 & 609 & 1.12 & 465 \\
\hline .08 & 297 & .43 & .56084 & .78 & 700 & 1.13 & 538 \\
\hline .09 & 453 & .44 & 201 & .79 & 790 & 1.14 & 610 \\
\hline 10 & 609 & .45 & 318 & .80 & 880 & 1.15 & 682 \\
\hline 11 & 763 & .46 & 433 & .81 & 969 & 1.16 & 754 \\
\hline 12 & 916 & .47 & 548 & .82 & .60057 & 1.17 & 825 \\
\hline 13 & .52068 & .48 & 662 & .83 & 145 & 1.18 & 896 \\
\hline 14 & 219 & .49 & 774 & .84 & 233 & 1.19 & 966 \\
\hline 15 & 368 & .50 & 886 & .85 & 319 & 1.20 & .63036 \\
\hline 16 & 516 & .51 & 997 & .86 & 406 & 1.21 & 106 \\
\hline 17 & 663 & .52 & .57107 & .87 & 491 & 1.22 & 175 \\
\hline 18 & 809 & .53 & 217 & .88 & 576 & 1.23 & 244 \\
\hline 19 & 954 & .54 & 325 & .89 & 661 & 1.24 & 313 \\
\hline 20 & .53097 & .55 & 433 & .90 & 745 & 1.25 & 381 \\
\hline 21 & 239 & .56 & 540 & .91 & 828 & 1.26 & 448 \\
\hline .22 & 380 & .57 & 646 & .92 & 911 & 1.27 & 516 \\
\hline .23 & 520 & .58 & 751 & .93 & 994 & 1.28 & 583 \\
\hline .24 & 659 & .59 & 855 & .94 & .61075 & 1.29 & 649 \\
\hline .25 & 796 & .60 & 959 & .95 & 157 & 1. 30 & 716 \\
\hline 26 & 932 & .61 & .58062 & .96 & 237 & 1. 31 & 781 \\
\hline .27 & .54068 & .62 & 164 & .97 & 318 & 1.32 & 847 \\
\hline 28 & 202 & $.63^{\circ}$ & 265 & .98 & 398 & 1.33 & 912 \\
\hline 29 & 335 & .64 & 365 & .99 & 477 & 1.34 & 977 \\
\hline 30 & 466 & .65 & 465 & 1.00 & 556 & 1.35 & 64041 \\
\hline 31 & 597 & .66 & 564 & 1.01 & 634 & 1. 36 & 106 \\
\hline 32 & 727 & .67 & 663 & 1.02 & 712 & 1. 37 & 169 \\
\hline 33 & 855 & .68 & 760 & 1.03 & 789 & 1. 38 & 233 \\
\hline 34 & 983 & .69 & 857 & 1.04 & 866 & 1. 39 . & 296 \\
\hline 35 & .55109 & .70 & 954 & 1.05 & 943 & 1.40 & 359 \\
\hline
\end{tabular}

Reprinted with the permission of Dr. J. Stuart Hunter, Editor of "Technometrics".

From the sample we evaluate $y=\ln A-\ln G$, and from the table we read or interpolate $\eta \rho$. Dividing the value of $\eta \rho(=y \rho)$ by $y$ we thus obtain the maximum likelihood estimate of $\rho=\alpha+1$, then from the second equation in (3.5) the estimate of $\lambda$. We shall write these estimates as $\hat{\rho}=\hat{\alpha}+1$, and $\hat{\lambda}$, respectively. The large sample variances of $\hat{\lambda}$ and $\hat{\alpha}$ are [Fisher 1922]

$$
\begin{aligned}
& \operatorname{var} \hat{\lambda} \simeq \frac{\lambda^{2}}{2 N} \\
& \operatorname{var} \hat{\alpha} \simeq \frac{1}{N}\left[\psi^{\prime}(1+\alpha)-\frac{1}{\alpha}+\frac{1}{2 \alpha^{2}}\right]^{-1},
\end{aligned}
$$

where $\psi^{\prime}(x)=d \psi(x) / d x$. The function $\psi^{\prime}(x)$ is tabulated in Davis (1935). An abstract of this table is presented in table 2 . If $\alpha$ is also large

$$
\operatorname{var} \hat{\alpha} \cong \frac{6}{N}\left(\alpha^{3}+\frac{1}{5} \alpha\right)
$$

To decide whether $q$ or $p=1 / q$ is to be assumed a gamma variate in a given situation we calculate the maximized likelihoods with $x=q$ and $p$. Let $L_{1}$ and $L_{2}$ be the values of $L(\hat{\alpha}, \hat{\lambda})$ corresponding to $x=q$ and $x=p$ respectively. If $L_{1}>L_{2}$ we decide that $q$ is a gamma variate; otherwise that $p$ is. Ordinarily a graphical criterion will suffice. For 
TABLE 2 .

\begin{tabular}{cccccc}
\hline$x$ & $\psi(x)$ & $\psi^{\prime}(x)$ & $x$ & $\psi(x)$ & $\psi^{\prime}(x)$ \\
\hline 0.1 & -10.42375 & 101.43330 & 1.1 & -0.42375 & 1.43330 \\
0.2 & -5.28904 & 26.26738 & 1.2 & -0.28904 & 1.26738 \\
0.3 & -3.50252 & 12.24536 & 1.3 & -0.16919 & 1.13425 \\
0.4 & -2.56138 & 7.27536 & 1.4 & -0.06138 & 1.02536 \\
0.5 & -1.96351 & 4.93480 & 1.5 & 0.03649 & 0.93480 \\
0.6 & -1.54062 & -3.63621 & 1.6 & 0.12605 & 0.85843 \\
0.7 & -1.22002 & 2.83405 & 1.7 & 0.20855 & 0.79323 \\
0.8 & -0.96501 & 2.29947 & 1.8 & 0.28499 & 0.73697 \\
0.9 & -0.75493 & 1.92254 & 1.9 & 0.35618 & 0.68797 \\
1.0 & -0.57722 & 1.64493 & 2.0 & 0.42278 & 0.64493 \\
\hline
\end{tabular}

Reproduced with the permission of Principia Press of Trinity University, San Antonio, Texas, Publishers.

example, we may proceed in the following manner. Let $F^{*}(x)$ denote the proportion of values of $Q$ exceeding $x$. Plot $F^{*}(x)$ versus $x$ on a normal probability paper with values of $x$ on the vertical axis increasing upward and values of $F^{*}(x)$ on the horizontal axis increasing from left to right. If the graph is concave (convex) toward the axes then $q(p)$ will be considered a gamma variate.

A quick but inefficient way of estimating $\alpha$ and $\lambda$ is to equate the first two moments (or logarithmic moments, in case the data are recorded in decibels) to the corresponding moments (or logarithmic moments) of (3.1).

The theoretical mean and variance of $\phi(x)$ are, respectively

so that

$$
m=\frac{\alpha+1}{\lambda}, \quad v=\frac{\alpha+1}{\lambda^{2}}
$$

$$
\lambda=\frac{m}{v}, \quad \alpha=\frac{m^{2}}{v}-1 .
$$

The sample estimates of $m$ and $v$ can then be used in these equations to estimate $\lambda$ and $\alpha$. If the value of $\alpha$ is small, say less than 5, this method, as shown by Fisher (1922), is extremely inefficient; hence almost always the estimation should be carried out by the method of maximum likelihood rather than by the method of moments.

Since power is usually measured in decibels, it is convenient to make rough estimates of $\alpha$ and $\lambda$ directly from such measurements. In particular if it is assumed that $x$ has a gamma distribution, and the mean and variance of the random variable

$$
\xi=10 \log _{10} x
$$

are $\xi^{\prime}$ and $\sigma_{\xi}^{2}$, then we have the relations

$$
\begin{aligned}
& \sigma_{\xi}^{2}=\left(10 \log _{10} e\right)^{2} \psi^{\prime}(1+\alpha), \\
& \bar{\xi}=\left(10 \log _{10} e\right)\left[\psi(1+\alpha)-\log _{e} \lambda\right],
\end{aligned}
$$

where $\psi(x)$ and $\psi^{\prime}(x)$ are defined earlier. The procedure for estimating parameters is a simple one:

1. Solve for $\alpha$ from the equation involving $\sigma_{\xi}^{2}$.

2 . Solve for $\lambda$, with the known $\alpha$, from the equation involving $\bar{\xi}$.

Concerning the efficiency of this estimation procedure, the same general remarks apply here as to the estimation procedure based on the moments of $x$.

\subsection{An Example}

In the following table we present 902 values of the hourly median power, expressed in decibels, received over the NBS path No. 55 from Detroit, Mich., to Hudson, Ohio: $\left(Q=10 \log _{10} q, f=\right.$ frequency of occurrence). The data correspond to the months of May through October 1950, to the time-period $6 \mathrm{pm}$ to midnight, and to the frequency of transmission 93.6 Mc/s. The decibel values are rounded to the nearest integer.

TABLE A.

\begin{tabular}{lrlllrll}
\hline $\mathrm{Q}$ & $\mathrm{f}$ & $\mathrm{Q}$ & $\mathrm{f}$ & $\mathrm{Q}$ & $\mathrm{f}$ & $\mathrm{Q}$ & $\mathrm{f}$ \\
\hline 1 & 0 & 13 & 67 & 25 & 15 & 37 & 3 \\
2 & 26 & 14 & 38 & 26 & 13 & 33 & 2 \\
3 & 5 & 15 & 24 & 27 & 7 & 39 & 6 \\
4 & 8 & 16 & 58 & 28 & 17 & 40 & 2 \\
5 & 9 & 17 & 33 & 29 & 5 & 41 & 1 \\
6 & 22 & 18 & 54 & 30 & 12 & 42 & 3 \\
7 & 27 & 19 & 38 & 31 & 9 & 43 & 1 \\
8 & 36 & 20 & 41 & 32 & 10 & 44 & 1 \\
9 & 27 & 21 & 25 & 33 & 4 & 45 & 2 \\
10 & 46 & 22 & 34 & 34 & 6 & 46 & 0 \\
11 & 68 & 23 & 20 & 35 & 5 & 47 & 0 \\
12 & 48 & 24 & 21 & 36 & 2 & 48 & 1 \\
\hline
\end{tabular}

Let $F^{*}(x)$ denote the proportion of values of $Q$ exceeding $x$. Then

$$
F^{*}(j+0.5)=1-\sum_{k=1}^{j} f_{k} / N, j=1,2, \ldots, 48,
$$

where $f_{k}$ is the frequency of the value $Q=k$. We note that the argument of $F^{*}$ is to be taken $j+0.5$ rather than $j$, as the integral value $j$ of $Q$ in the table is actually the midpoint of the half-open interval $(j-0.5, j+0.5)$. A visual check of the graph of $F^{*}(x)$ on the normal probability paper indicated that $p=1 / q$ rather than $q$ should be assumed to be a gamma variate. A test based on the likelihood ratio seemed unnecessary. To estimate $\alpha$ and $\lambda$ by the method of maximum likelihood we calculate the arithmetic mean, $A_{p}$, and the geometric mean, $G_{p}$, of $p$. These are

$$
A_{p}=0.07565, G_{p}=0.02118 \text {. }
$$

Thus $\eta=\ln A_{p}-\ln G_{p}=1.2729$, and interpolation in table 1 gives $\eta \rho=0.6354$. Finally, $\hat{\rho}=\hat{\alpha}+1 \cong 0.50, \hat{\lambda}$ $=\hat{\rho} / A_{p} \cong 6.6$. From (3.6), with $\lambda$ and $\alpha$ replaced by $\hat{\lambda}$ and $\hat{\alpha}$, the estimates of the variance of $\hat{\lambda}$ and $\hat{\alpha}$ are calculated to be

\section{Estimate of $\operatorname{var} \hat{\lambda} \cong 0.024$,}

Estimate of var $\hat{a} \simeq 0.00012$.

For the best fitting lognormal distribution for $q$, i.e., for the best fitting normal distribution for $Q$, we calculate the maximum likelihood estimates of the mean and variance of $Q$ to be

$$
\bar{Q}=\frac{\Sigma f Q}{N}=16.74, \hat{\sigma}^{2}=\frac{\Sigma f Q^{2}}{N}-\bar{Q}^{2}=72.35,
$$




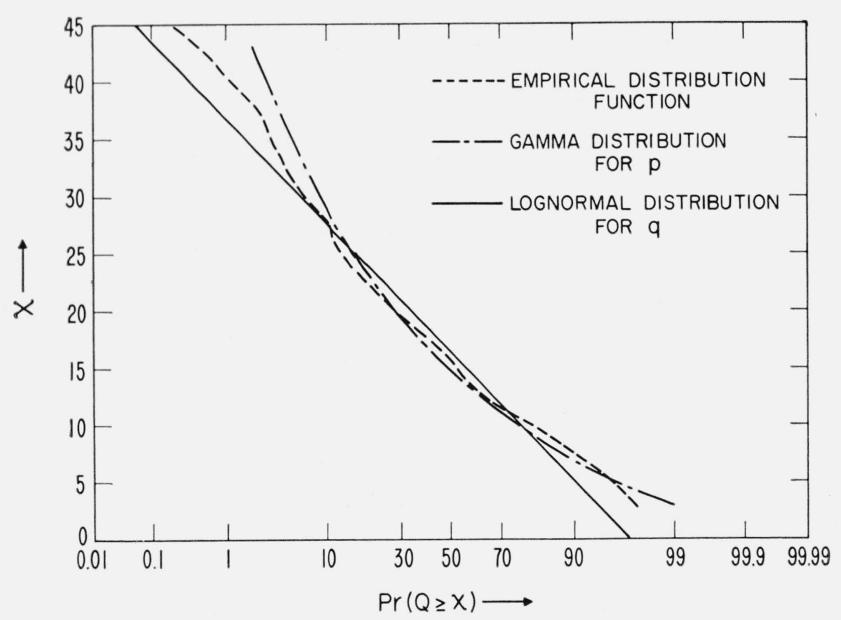

FIgure 5. The empirical distribution function of $\mathrm{q}$, together with the best fitting gamma distribution for $1 / \mathrm{q}$ and the best fitting lognormal distribution for q, for the data in section 3.1.

respectively. The graphs of $F^{*}(x)$, and the best fitting gamma and lognormal distributions are given in figure 5 .

To see whether the gamma distribution for $p$ gives a better fit to the data than the lognormal for $q$, we calculate the maximized likelihoods in both cases. The logarithm (on base 10) of the maximized likelihood under the hypothesis of gamma distribution for $p$ is +703 , while that under the lognormal distribution for $q$ is -1398 . Thus, in the present situation, the hypothesis that $p$ is a gamma variate is decidedly preferred over the other alternative.

\section{Long-Term Distribution of Instantaneous Power}

When incoherent scatter is the dominant mode of radio wave propagation, it has been observed that over short periods of time the instantaneous received power, $z$, is Rayleigh distributed. If the mean power over this period be denoted by $q$, the probability density function of $z$ is given by

$$
f(z \mid q)=q^{-1} e^{-z / q}, \quad z \geq 0, q>0 .
$$

This can also be written, with $p=1 / q$, as

$$
f_{1}(z \mid p)=p e^{-p z}, \quad z \geq 0, p>0 .
$$

If it is assumed that $q$ itself has the probability density function $h(q)$, the (long-term) probability density function of $z$ will be given by

$$
f(z)=\int_{0}^{\infty} f(z \mid q) h(q) d q .
$$

If now $q$ or $p$ is assumed to have a gamma probability density function $\phi(x)$ given in (3.1), we have in the one case

$$
f(z)=\int_{0}^{\infty} f(z \mid q) \phi(q) d q
$$

and in the other

$$
f_{1}(z)=\int_{0}^{\infty} f_{1}(z \mid p) \phi(p) d p .
$$

We will first consider the case when $p$ is a gamma variate.

Situation 1. If $p$ has the probability density function $\phi(x)$, then

$$
\begin{aligned}
f_{1}(z) & =\frac{\lambda^{\alpha+1}}{\Gamma(\alpha+1)} \int_{0}^{\infty} e^{-p(\lambda+z)} p^{\alpha+1} d p \\
& =(\alpha+1) \lambda^{\alpha+1}(\lambda+z)^{-\alpha-2} .
\end{aligned}
$$

The tail probability, i.e., the probability that the power exceeds a given level $z$,

$$
F_{1}(z)=\int_{z}^{\infty} f_{1}(x) d x=\left(1+\frac{z}{\lambda}\right)^{-\alpha-1} .
$$

For large $z / \lambda$ this behaves like

$$
F_{1}(z) \sim(\lambda / z)^{\alpha+1},
$$

and for small $z / \lambda$,

$$
F_{1}(z)=1-(\alpha+1)(z / \lambda)+O\left[(z / \lambda)^{2}\right],
$$

where $O(x)$ signifies a quantity of the order of magnitude of $x$. The $r$ th moment of the distribution of $z$ is nonexistent if $\alpha \leq r-1$. In particular, if $-1<\alpha \leq 0$, no moment (except the zeroth moment which is always equal to unity) exists. If $\alpha+1>r$,

$$
E(z+\lambda)^{r}=(\alpha+1) \lambda^{\alpha+1} \int_{0}^{\infty}(z+\lambda)^{r-\alpha-2} d z=\frac{(\alpha+1) \lambda^{r}}{(\alpha+1-r)} .
$$

Thus, if $\alpha>1$, the mean and variance of $z$ are

$$
E_{z}=\frac{\lambda}{\alpha}, \operatorname{var} z=\frac{(\alpha+1) \lambda^{2}}{\alpha^{2}(\alpha-1)} .
$$

Let $0<\beta<1$. The $100 \beta$ percentile, $z_{\beta}$, of $F_{1}(z)$ is given by

$$
z_{\beta}=\left[\left(\frac{1}{\beta}\right)^{\frac{1}{1+\alpha}}-1\right] \lambda .
$$

In particular, the median

$$
z_{0.5}=\left[2^{\frac{1}{\alpha+1}}-1\right] \lambda .
$$

In figure $6, F_{1}(z)$ is plotted on the normal probability paper against $y=10 \log _{10}\left(z / z_{0.5}\right)$, for selected values of $\alpha$.

Situation 2 . If $q$ has the probability density function $\phi(x)$, then 


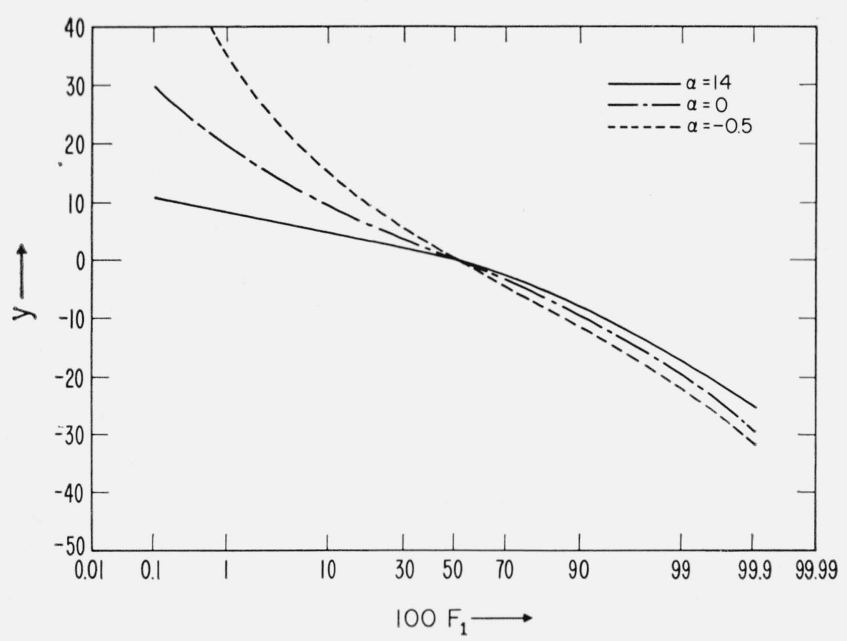

Figure 6. $100 \mathrm{~F}_{1}(\mathrm{z})$ versus $\mathrm{y}=10\left(\log \mathrm{z}-\log \mathrm{z}_{0.5}\right), \mathrm{z}_{0.5}=$ median of $\mathrm{z}$.

$$
f(z)=\frac{\lambda^{\alpha+1}}{\Gamma(\alpha+1)} \int_{0}^{\infty} q^{\alpha-1} \exp (-\lambda q-z / q) d q .
$$

A change of variate $q=(z / \lambda)^{\frac{1}{2}} e^{\theta}$ converts this into

$$
\begin{aligned}
f(z) & =\frac{\lambda(z \lambda)^{\alpha / 2}}{\Gamma(\alpha+1)} \int_{-\infty}^{\infty} \exp (\alpha \theta-2 \sqrt{z \lambda} \cosh \theta) d \theta \\
& =\frac{2 \lambda(z \lambda)^{\alpha / 2}}{\Gamma(\alpha+1)} \int_{0}^{\infty} \cosh \alpha \theta \exp \mid(-2 \sqrt{z \lambda} \cosh \theta) d \theta .
\end{aligned}
$$

Using the Bessel function formula

$$
K_{\alpha}(x)=\int_{0}^{\infty} \cosh \alpha \theta \exp (-x \cosh \theta) d \theta
$$

we have

$$
f(z)=\frac{2 \lambda(z \lambda)^{\alpha / 2}}{\Gamma(\alpha+1)} K_{\alpha}(2 \sqrt{z \lambda})
$$

The tail probability, i.e., the probability that the power exceeds a given level $z$,

$$
F(z)=\int_{z}^{\infty} f(x) d x
$$

is found by the same methods to be

$$
F(z)=\frac{2(z \lambda)^{\frac{\alpha+1}{2}}}{\Gamma(\alpha+1)} K_{\alpha+1}(2 \sqrt{z \lambda}) .
$$

For large $z \lambda$ this behaves like

$$
\left.F(z) \sim \frac{\sqrt{\pi}}{\Gamma(\alpha+1)}(z \lambda)^{\frac{2 \alpha+1}{4}} e\right)^{-2 \sqrt{2 \lambda}}
$$

and is seen to fall off rather slowly when $z \lambda$ is of the order of unity. For small $z \lambda$, when $\alpha$ is not an integer,

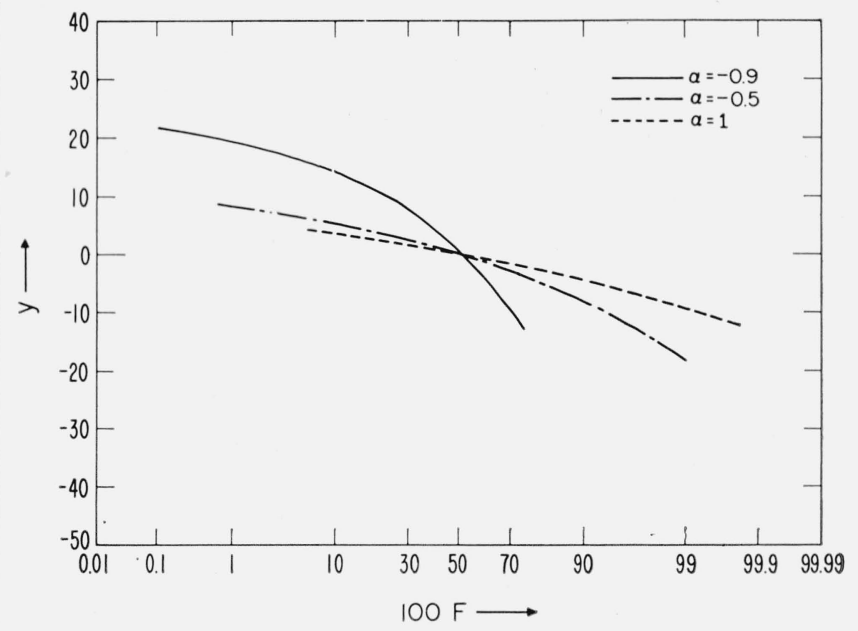

FIGURE 7. $100 \mathrm{~F}(\mathrm{z})$ versus $\mathrm{y}=10\left(\log \mathrm{z}-\log \mathrm{z}_{0.5}\right), \mathrm{z}_{0.5}=$ median of $\mathrm{z}$.

$$
F(z)=1-\frac{\Gamma(-\alpha)}{\Gamma(\alpha+2)}(z \lambda)^{1+\alpha}-\frac{z \lambda}{\alpha}+0(z \lambda),
$$

where $\theta(x)$ signifies a quantity which tends to zero more rapidly than $x$ when $x$ tends to zero. The moments of the distribution of $z$ are easily evaluated to be, for $r=0,1,2$, . . .,

$$
\mu_{r}=\int_{0}^{\infty} z^{r} f(z) d z=\frac{r ! \Gamma(\alpha+r+1)}{\Gamma(\alpha+1)} \frac{1}{\lambda^{r}} .
$$

In table 3 we give values of $F(z)$ as a function of the dimensionless variable $x=2 \sqrt{z \lambda}$ for $\alpha=-0.9(0.1)$ 1.0, $x=0(0.1) 5.0$ and for $\alpha=-0.9(0.1) 0.0$ with $x=0(0.01) .1$. There may be a roundoff error of one in the last place. For values of $\alpha$ exceeding 1.0 we may use the recurrence formula for $K_{m}(x)$ :

$$
K_{m+1}(x)=\frac{2 m}{x} K_{m}(x)+K_{m-1}(x) .
$$

If we denote by $F_{\alpha}(x)$ the tail probability as a function of $x$ and $\alpha$, i.e.,

$$
F_{\alpha}(x)=\frac{x^{\alpha+1}}{2^{\alpha} \Gamma(\alpha+1)} K_{\alpha+1}(x),
$$

then the use of the relations between $K$ 's leads to

$$
F_{\alpha+1}(x)=F_{\alpha}(x)+\frac{x^{2}}{4 \alpha(\alpha+1)} F_{\alpha-1}(x) .
$$

In figure $7 F(z)$ is plotted on the normal probability paper against $y=10 \log _{10}\left(z / z_{0.5}\right)$, where $z_{0.5}$ is the median of the distribution of $z$.

\section{Discussion}

To keep the mathematical reasoning uncluttered with nonmathematical considerations we have glided 
Table 3.

\begin{tabular}{|c|c|c|c|c|c|c|c|c|c|c|}
\hline $\mathrm{x}$ & $a=-0.9$ & -0.8 & -0.7 & .0 .6 & -0.5 & -0.4 & -0.3 & -0.2 & -0.1 & 0.0 \\
\hline 0.01 & 0.6107 & 0.8477 & 0.9398 & 0.9758 & 0.9900 & 0.9958 & 0.9981 & 0.9991 & 0.9995 & 0.9997 \\
\hline 0.02 & 0.5529 & 0.7991 & 0.9089 & 0.9580 & 0.9802 & 0.9904 & 0.9951 & 0.9974 & 0.9985 & 0.9991 \\
\hline 0.03 & 0.5152 & 0.7639 & 0.8839 & 0.9421 & 0.9704 & 0.9845 & 0.9915 & 0.9952 & 0.9971 & 0.9981 \\
\hline 0.04 & 0.4866 & 0.7352 & 0.8622 & 0.9272 & 0.9608 & 0.9783 & 0.9876 & 0.9926 & 0.9953 & 0.9969 \\
\hline 0.05 & 0.4633 & 0.7107 & 0.8427 & 0.9133 & 0.9512 & 0.9719 & 0.9833 & 0.9896 & 0.9933 & 0.9955 \\
\hline 0.06 & 0.4435 & 0.6890 & 0.8247 & 0.8999 & 0.9418 & 0.9653 & 0.9787 & 0.9865 & 0.9910 & 0.9938 \\
\hline 0.07 & 0.4262 & 0.6695 & 0.8081 & 0.8871 & 0.9324 & 0.9586 & 0.9739 & 0.9830 & 0.9885 & 0.9920 \\
\hline 0.08 & 0.4109 & 0.6516 & 0.7924 & 0.8747 & 0.9231 & 0.9518. & 0.9690 & 0.9794 & 0.9859 & 0.9899 \\
\hline 0.09 & 0.3970 & 0.6351 & 0.7775 & 0.8627 & 0.9139 & 0.9449 & 0.9638 & 0.9756 & 0.9830 & 0.9877 \\
\hline 0.1 & 0.3844 & 0.6198 & 0.7634 & 0.8511 & 0.9048 & 0.9380 & 0.9586 & 0.9716 & 0.9799 & 0.9854 \\
\hline 0.2 & 0.2959 & 0.5035 & 0.6482 & 0.7488 & 0.8187 & 0.8675 & 0.9016 & 0.9257 & 0.9429 & 0.9552 \\
\hline 0.3 & 0.2407 & 0.4234 & $0.56,09$ & 0.6639 & 0.7408 & 0.7983 & 0.8414 & 0.8737 & 0.8982 & 0.9168 \\
\hline 0.4 & 0.2009 & 0.3621 & 0.4901 & 0.5910 & 0.6703 & 0.7325 & 0.7813 & 0.8196 & 0.8498 & 0.8737 \\
\hline 0.5 & 0.1702 & 0.3127 & 0.4307 & 0.5275 & 0.6065 & 0.6708 & 0.7231 & 0.7655 & 0.8000 & 0.8282 \\
\hline 0.6 & 0.1457 & 0.2720 & 0.3801 & 0.4717 & 0.5488 & 0.6134 & 0.6675 & 0.7126 & 0.7502 & 0.7817 \\
\hline 0.7 & 0.1256 & 0.2378 & 0.3365 & 0.4224 & 0.4966 & 0.5603 & 0.6149 & 0.6615 & 0.7013 & 0.7352 \\
\hline 0.8 & 0.1089 & 0.2086 & 0.2985 & 0.3786 & 0.4493 & 0.5114 & 0.5656 & 0.6128 & 0.6538 & 0.6894 \\
\hline 0.9 & 0.0948 & 0.1836 & 0.2654 & 0.3397 & 0.4066 & 0.4664 & 0.5195 & 0.5666 & 0.6082 & 0.6449 \\
\hline 1.0 & 0.0829 & 0.1620 & 0.2363 & 0.3050 & 0.3679 & 0.4251 & 0.4767 & 0.5231 & 0.5647 & 0.6019 \\
\hline 1.1 & 0.0726 & 0.1433 & 0.2106 & 0.2740 & 0.3329 & 0.3872 & 0.4370 & 0.4823 & 0.5235 & 0.5607 \\
\hline 1. 2 & 0.0638 & 0.1269 & 0.1880 & 0.2462 & 0.3012 & 0.3526 & 0.4002 & 0.4442 & 0.4846 & 0.5215 \\
\hline 1. 3 & 0.0562 & 0.1125 & 0.1679 & 0.2214 & 0.2725 & 0.3209 & 0.3663 & 0.4086 & 0.4480 & 0.4843 \\
\hline 1. 4 & 0.0496 & 0.0999 & 0.1501 & 0.1992 & 0.2466 & 0.2920 & 0.3350 & 0.3756 & 0.4137 & 0.4492 \\
\hline 1. 5 & 0.0438 & 0.0889 & 0.1343 & 0.1792 & 0.2231 & 0.2656 & 0.3063 & 0.3450 & 0.3816 & 0.4161 \\
\hline 1. 6 & 0.0387 & 0.0791 & 0.1202 & 0.1613 & 0.2019 & 0.2415 & 0.2798 & 0.3166 & 0.3517 & 0.3850 \\
\hline 1. 7 & 0.0343 & 0.0705 & 0.1077 & 0.1452 & 0.1827 & 0.2196 & 0.2555 & 0.2904 & 0.3239 & 0.3559 \\
\hline 1. 8 & 0.0304 & 0.0628 & 0.0965 & 0.1308 & 0.1653 & 0.1996 & 0.2333 & 0.2661 & 0.2980 & 0.3287 \\
\hline 1. 9 & 0.0270 & 0.0560 & 0.0865 & 0.1178 & 0.1496 & 0.1814 & 0.2128 & 0.2438 & 0.2740 & 0.3034 \\
\hline
\end{tabular}

TABLE $3 a$.

\begin{tabular}{|c|c|c|c|c|c|c|c|c|c|c|c|}
\hline $\mathrm{x}$ & $a=$ & -0.9 & -0.8 & -0.7 & -0.6 & -0.5 & -0.4 & -0.3 & -0.2 & -0.1 & 0.0 \\
\hline 2. 0 & & 0.0240 & 0.0500 & 0.0776 & 0.1061 & 0.1353 & 0.1648 & 0.1942 & 0.2232 & 0.2518 & 0.2797 \\
\hline 2.1 & & 0.0213 & 0.0447 & 0.0696 & 0.0957 & 0.1225 & 0.1497 & 0.1770 & 0.2043 & 0.2313 & 0.2578 \\
\hline 2. 2 & & 0.0190 & 0.0399 & 0.0625 & 0.0862 & 0.1108 & 0.1360 & 0.1614 & 0.1869 & 0.2123 & 0.2374 \\
\hline 2. 3 & & 0.0169 & 0.0357 & 0.0561 & 0.0777 & 0.1003 & 0.1235 & 0.1471 & 0.1709 & 0.1948 & 0.2185 \\
\hline 2. 4 & & 0.0151 & 0.0320 & 0.0504 & 0.0701 & 0.0907 & 0.1121 & 0.1340 & 0.1562 & 0.1786 & 0.2009 \\
\hline 2. 5 & & 0.0134 & 0.0286 & 0.0453 & 0.0632 & 0.0821 & 0.1018 & 0.1221 & 0.1428 & 0.1637 & 0.1847 \\
\hline 2.6 & & 0.0120 & 0.0256 & 0.0407 & 0.0570 & 0.0743 & 0.0924 & 0.1112 & 0.1304 & 0.1500 & 0.1697 \\
\hline 2. 7 & & 0.0107 & 0.0229 & 0.0366 & 0.0514 & 0.0672 & 0.0839 & 0.1012 & 0.1191 & 0.1374 & 0.1559 \\
\hline 2. 8 & & 0.0095 & 0.0205 & 0.0329 & 0.0463 & 0.0608 & 0.0761 & 0.0922 & 0.1088 & 0.1258 & 0.1431 \\
\hline 2. 9 & & 0.0085 & 0.0184 & 0.0295 & 0.0418 & 0.0550 & 0.0691 & 0.0839 & 0.0993 & 0.1151 & 0.1313 \\
\hline 3. 0 & & 0.0076 & 0.0165 & 0.0266 & 0.0377 & 0.0498 & 0.0627 & 0.0763 & 0.0906 & 0.1053 & 0.1205 \\
\hline 3.1 & & 0.0068 & 0.0148 & 0.0239 & 0.0340 & 0.0450 & 0.0569 & 0.0695 & 0.0826 & 0.0963 & 0.1105 \\
\hline 3. 2 & & 0.0061 & 0.0133 & 0.0215 & 0.0307 & 0.0408 & 0.0516 & 0.0632 & 0.0754 & 0.0881 & 0.1013 \\
\hline 3.3 & & 0.0054 & 0.0119 & 0.0194 & 0.0277 & 0.0369 & 0.0468 & 0.0575 & 0.0687 & 0.0805 & 0.0928 \\
\hline 3. 4 & & 0.0049 & 0.0107 & 0.0174 & 0.0250 & 0.0334 & 0.0425 & 0.0523 & 0.0627 & 0.0736 & 0.0850 \\
\hline 3.5 & & 0.0044 & 0.0096 & 0.0157 & 0.0226 & 0.0302 & 0.0385 & 0.0475 & 0.0571 & 0.0672 & 0.0778 \\
\hline 3.6 & & 0.0039 & 0.0086 & 0.0141 & 0.0204 & 0.0273 & 0.0350 & 0.0432 & 0.0521 & 0.0614 & 0.0713 \\
\hline 3.7 & & 0.0035 & 0.0077 & 0.0127 & 0.0184 & 0.0247 & 0.0317 & 0.0393 & 0.0474 & 0.0561 & 0.0652 \\
\hline 3. 8 & & 0.0031 & 0.0069 & 0.0114 & 0.0166 & 0.0224 & 0.0288 & 0.0357 & 0.0432 & 0.0512 & 0.0597 \\
\hline 3. 9 & & 0.0028 & 0.0062 & 0.0103 & 0.0150 & 0.0202 & 0.0261 & 0.0325 & 0.0394 & 0.0468 & 0.0546 \\
\hline 4. 0 & & 0.0025 & 0.0056 & 0.0093 & 0.0135 & 0.0183 & 0.0237 & 0.0295 & 0.0359 & 0.0427 & 0.0499 \\
\hline 4.1 & & 0.0023 & 0.0050 & 0.0084 & 0.0122 & 0.0166 & 0.0214 & 0.0268 & 0.0327 & 0.0389 & 0.0457 \\
\hline 4.2 & & 0.0020 & 0.0045 & 0.0075 & 0.0110 & 0.0150 & 0.0195 & 0.0244 & 0.0297 & 0.0355 & 0.0417 \\
\hline 4.3 & & 0.0018 & 0.0041 & 0.0068 & 0.0099 & 0.0136 & 0.0176 & 0.0221 & 0.0271 & 0.0324 & 0.0381 \\
\hline 4.4 & & 0.0016 & 0.0037 & 0.0061 & 0.0090 & 0.0123 & 0.0160 & 0.0201 & 0.0246 & 0.0296 & 0.0349 \\
\hline 4.5 & & 0.0015 & 0.0033 & 0.0055 & 0.0081 & 0.0111 & 0.0145 & 0.0183 & 0.0224 & 0.0270 & 0.0319 \\
\hline 4.6 & & 0.0013 & 0.0030 & 0.0050 & 0.0073 & 0.0101 & 0.0131 & 0.0166 & 0.0204 & 0.0246 & 0.0291 \\
\hline 4.7 & & 0.0012 & 0.0027 & 0.0045 & 0.0066 & 0.0091 & 0.0119 & 0.0151 & 0.0186 & 0.0224 & 0.0266 \\
\hline 4.8 & & 0.0011 & 0.0024 & 0.0040 & 0.0060 & 0.0082 & 0.0108 & 0.0137 & 0.0169 & 0.0204 & 0.0243 \\
\hline 4.9 & & 0.0009 & 0.0022 & 0.0036 & 0.0054 & 0.0074 & 0.0098 & 0.0124 & 0.0154 & 0.0186 & 0.0222 \\
\hline 5.0 & & 0.0009 & 0.0019 & 0.0033 & 0.0049 & 0.0067 & 0.0089 & 0.0113 & 0.0140 & 0.0170 & 0.0202 \\
\hline
\end{tabular}


TABLE $3 \mathrm{~b}$.

\begin{tabular}{|c|c|c|c|c|c|c|c|c|c|c|c|}
\hline $\mathrm{x}$ & $a=$ & 0.1 & 0.2 & 0.3 & 0.4 & 0.5 & 0.6 & 0.7 & 0.8 & 0.9 & 1.0 \\
\hline 0.1 & & 0.9890 & 0.9915 & 0.9932 & 0.9944 & 0.9953 & 0.9960 & 0.9965 & 0.9969 & 0.9973 & 0.9975 \\
\hline 0.2 & & 0.9642 & 0.9708 & 0.9758 & 0.9796 & 0.9825 & 0.9848 & 0.9866 & 0.9880 & 0.9893 & 0.9902 \\
\hline 0.3 & & 0.9311. & 0.9421 & 0.9508 & 0.9576 & 0.9631 & 0.9675 & 0.9711 & 0.9740 & 0.9765 & 0.9786 \\
\hline 0.4 & & 0.8928 & 0.9080 & 0.9203 & 0.9303 & 0.9384 & 0.9452 & 0.9508 & 0.9555 & 0.9595 & 0.9629 \\
\hline 0.5 & & 0.8513 & 0.8702 & 0.8859 & 0.8989 & 0.9098 & 0.9189 & 0.9267 & 0.9333 & 0.9389 & 0.9438 \\
\hline 0.6 & & 0.8081 & 0.8302 & 0.8489 & 0.8647 & 0.8781 & 0.8896 & 0.8994 & 0.9079 & 0.9152 & 0.9217 \\
\hline 0.7 & & 0.7642 & 0.7889 & 0.8102 & 0.8284 & 0.8442 & 0.8578 & 0.8697 & 0.8800 & 0.8891 & 0.8970 \\
\hline 0.8 & & 0.7203 & 0.7472 & 0.7706 & 0.7910 & 0.8088 & 0.8244 & 0.8381 & 0.8502 & 0.8609 & 0.8703 \\
\hline 0.9 & & 0.6772 & 0.7057 & 0.7308 & 0.7529 & 0.7725 & 0.7898 & 0.8052 & 0.8189 & 0.8311 & 0.8420 \\
\hline 1.0 & & 0.6351 & 0.6647 & 0.6911 & 0.7147 & 0.7358 & 0.7546 & 0.7714 & 0.7866 & 0.8002 & 0.8124 \\
\hline 1.1 & & 0.5944 & 0.6247 & 0.6521 & 0.6768 & 0.6990 & 0.7191 & 0.7372 & 0.7536 & 0.7685 & 0.7819 \\
\hline 1.2 & & 0.5553 & 0.5860 & 0.6140 & 0.6395 & 0.6626 & 0.6837 & 0.7029 & 0.7204 & 0.7363 & 0.7508 \\
\hline 1.3 & & 0.5178 & 0.5487 & 0.5770 & 0.6030 & 0.6268 & 0.6487 & 0.6687 & 0.6871 & 0.7039 & 0.7194 \\
\hline 1.4 & & 0.4822 & 0.5129 & 0.5413 & 0.5676 & 0.5918 & 0.6142 & 0.6349 & 0.6540 & 0.6717 & 0.6880 \\
\hline 1.5 & & 0.4484 & 0.4787 & 0.5070 & 0.5333 & 0.5578 & 0.5806 & 0.6018 & 0.6214 & 0.6397 & 0.6566 \\
\hline 1.6 & & 0.4165 & 0.4462 & 0.4742 & 0.5004 & 0.5249 & 0.5479 & 0.5694 & 0.5894 & 0.6081 & 0.6256 \\
\hline 1.7 & & 0.3865 & 0.4155 & 0.4429 & 0.4688 & 0.4932 & 0.5162 & 0.5378 & 0.5581 & 0.5772 & 0.5951 \\
\hline 1.8 & & 0.3582 & 0.3864 & 0.4132 & 0.4387 & 0.4628 & 0.4857 & 0.5073 & 0.5277 & 0.5470 & 0.5651 \\
\hline 1.9 & & 0.3317 & 0.3589 & 0.3850 & 0.5000 & 0.4337 & 0.4564 & 0.4779 & 0.4983 & 0.5176 & 0.5359 \\
\hline 2.0 & & 0.3069 & 0.3331 & 0.3584 & 0.3827 & 0.4060 & 0.4283 & 0.4496 & 0.4698 & 0.4892 & 0.5075 \\
\hline 2.1 & & 0.2837 & 0.3089 & 0.3333 & 0.3569 & 0.3796 & 0.4015 & 0.4224 & 0.4425 & 0.4617 & 0.4800 \\
\hline 2.2 & & 0.2620 & 0.2862 & 0.3097 & 0.3325 & 0.3546 & 0.3759 & 0.3965 & 0.4162 & 0.4352 & 0.4534 \\
\hline 2. 3 & & 0.2419 & 0.2649 & 0.2875 & 0.3095 & 0.3309 & 0.3516 & 0.3717 & 0.3911 & 0.4098 & 0.4278 \\
\hline 2. 4 & & 0.2231 & 0.2451 & 0.2666 & 0.2878 & 0.3084 & 0.3286 & 0.3481 & 0.3671 & 0.3854 & 0.4032 \\
\hline 2.5 & & 0.2057 & 0.2265 & 0.2471 & 0.2674 & 0.2873 & 0.3068 & 0.3257 & 0.3442 & 0.3622 & 0.3796 \\
\hline 2.6 & & 0.1895 & 0.2093 & 0.2289 & 0.2483 & 0.2674 & 0.2861 & 0.3045 & 0.3225 & 0.3400 & 0.3570 \\
\hline 2.7 & & 0.1745 & 0.1932 & 0.2119 & 0.2304 & 0.2487 & 0.2667 & 0.2844 & 0.3018 & 0.3188 & 0.3354 \\
\hline 2.8 & & 0.1607 & 0.1783 & 0.1960 & 0.2136 & 0.2311 & 0.2484 & 0.2655 & 0.2822 & 0.2987 & 0.3149 \\
\hline 2. 9 & & 0.1478 & 0.1644 & 0.1812 & 0.1979 & 0.2146 & 0.2312 & 0.2476 & 0.2637 & 0.2797 & 0.2954 \\
\hline 3. 0 & & 0.1359 & 0.1516 & 0.1674 & 0.1833 & 0.1991 & 0.2150 & 0.2307 & 0.2463 & 0.2616 & 0.2768 \\
\hline 3.1 & & 0.1249 & 0.1397 & 0.1546 & 0.1696 & 0.1847 & 0.1998 & 0.2148 & 0.2298 & 0.2446 & 0.2592 \\
\hline 3.2 & & 0.1148 & 0.1286 & 0.1427 & 0.1569 & 0.1712 & 0.1856 & 0.1999 & 0.2142 & 0.2284 & 0.2425 \\
\hline
\end{tabular}

TABLE 3c.

\begin{tabular}{ccccccccccc}
\hline $\mathrm{x} \quad \mathrm{a}=$ & 0.1 & 0.2 & 0.3 & 0.4 & 0.5 & 0.6 & 0.7 & 0.8 & 0.9 & 1.0 \\
\hline 3.3 & 0.1054 & 0.1184 & 0.1316 & 0.1450 & 0.1586 & 0.1722 & 0.1859 & 0.1996 & 0.2132. & 0.2268 \\
3.4 & 0.0968 & 0.1089 & 0.1214 & 0.1340 & 0.1468 & 0.1598 & 0.1728 & 0.1856 & 0.1989 & 0.2119 \\
3.5 & 0.0888 & 0.1002 & 0.1119 & 0.1238 & 0.1359 & 0.1481 & 0.1605 & 0.1730 & 0.1854 & 0.1979 \\
3.6 & 0.0815 & 0.0921 & 0.1031 & 0.1143 & 0.1257 & 0.1373 & 0.1490 & 0.1608 & 0.1727 & 0.1847 \\
3.7 & 0.0748 & 0.0847 & 0.0949 & 0.1054 & 0.1162 & 0.1272 & 0.1383 & 0.1495 & 0.1608 & 0.1722 \\
3.8 & 0.0686 & 0.0778 & 0.0874 & 0.0973 & 0.1074 & 0.1177 & 0.1282 & 0.1389 & 0.1497 & 0.1605 \\
3.9 & 0.0628 & 0.0715 & 0.0804 & 0.0897 & 0.0992 & 0.1089 & 0.1189 & 0.1290 & 0.1392 & 0.1495 \\
4.0 & 0.0576 & 0.0656 & 0.0740 & 0.0826 & 0.0916 & 0.1008 & 0.1101 & 0.1197 & 0.1294 & 0.1392 \\
4.1 & 0.0528 & 0.0602 & 0.0680 & 0.0761 & 0.0845 & 0.0931 & 0.1020 & 0.1110 & 0.1202 & 0.1295 \\
4.2 & 0.0483 & 0.0553 & 0.0625 & 0.0701 & 0.0780 & 0.0861 & 0.0944 & 0.1029 & 0.1116 & 0.1205 \\
4.3 & 0.0443 & 0.0507 & 0.0575 & 0.0646 & 0.0719 & 0.0795 & 0.0874 & 0.0954 & 0.1036 & 0.1120 \\
4.4 & 0.0405 & 0.0465 & 0.0528 & 0.0594 & 0.0663 & 0.0734 & 0.0808 & 0.0884 & 0.0961 & 0.1041 \\
4.5 & 0.0371 & 0.0426 & 0.0485 & 0.0547 & 0.0611 & 0.0678 & 0.0747 & 0.0818 & 0.0892 & 0.0966 \\
4.6 & 0.0339 & 0.0391 & 0.0445 & 0.0503 & 0.0563 & 0.0625 & 0.0690 & 0.0757 & 0.0826 & 0.0897 \\
4.7 & 0.0310 & 0.0358 & 0.0409 & 0.0462 & 0.0518 & 0.0577 & 0.0638 & 0.0701 & 0.0766 & 0.0833 \\
4.8 & 0.0284 & 0.0328 & 0.0375 & 0.0425 & 0.0477 & 0.0532 & 0.0589 & 0.0648 & 0.0709 & 0.0772 \\
4.9 & 0.0260 & 0.0301 & 0.0344 & 0.0391 & 0.0439 & 0.0490 & 0.0544 & 0.0599 & 0.0657 & 0.0716 \\
5.0 & 0.0237 & 0.0275 & 0.0316 & 0.0359 & 0.0404 & 0.0452 & 0.0502 & 0.0554 & 0.0608 & 0.0664 \\
\hline
\end{tabular}


over a few points which need to be discussed more fully.

First, it may be mentioned that the observations on $z$ come as a time series. In this paper we have assumed that the (univariate) distribution function of $z$, hence of $q$, is time-invariant. For estimating the long-term distribution this assumption may be quite valid. However, if one wishes to study other characteristics of the data on $z$, such as the presence of diurnal and seasonal cycles, it would be necessary to use the methodology of time series analysis. This was not attempted in this paper.

Second, assuming that the distributions of $z$ and of $q$ are time-invariant, that the short-term distribution of $z$ is Rayleigh with mean $q$, and that the distribution of $q$ or $1 / q$ is either lognormal or gamma, the question still remains: prior to a contemplated experiment how can we say which distribution will apply? To answer this question we require considerably more understanding of the physics of scatter propagation, a detailed knowledge of important physical variables over a transmission path, and an elaborate investigation of various statistical hypotheses. Thus, at the present state of the art, this question cannot be answered. However, after the data are collected, a decision can be made based on either the likelihood ratio criterion or on some other "goodness-of-fit" test such as $\chi^{2}$ or Kolmogorov's tests.

Third, to insure independence between observations as required in section 3 for estimating $\alpha$ and $\lambda$ it is necessary to take values of $q$ sufficiently widely separated. This again may require a preliminary investigation of the autocorrelation function of $q$. In practice, it will not be difficult to decide how far apart these values are to be taken. We note that, if the values of $q$ constitute a random sample so will the values of $p$.

Fourth, we have not discussed the problem of estimating $\alpha$ and $\lambda$ as the parameters of $F(z)$ or $F_{1}(z)$. We have implicitly assumed that $\lambda$ and $\alpha$ will be estimated as the parameters of the gamma distribution from a sample of values of $q$. In prin- ciple, it is preferable to apply the method of maximum likelihood to the distribution of $z$ itself if the object is to obtain a distribution of $z$ (rather than that of q). However, in this case, the solution of likelihood equations seemed to be laborious, hence we have accepted the estimating procedure as outlined in section 3 .

Lastly, a more flexible approach to the entire problem is to assume that $x=\lambda q^{\beta}, \lambda>0, \beta \neq 0$, is a gamma variate. This was briefly mentioned at the end of section 2. In this case, we will have three parameters, $\lambda, \beta$, and $\alpha$, instead of two. The main difficulty, again, is in the estimation procedure. We hope to investigate this generalized model in the future.

We thank Mrs. Mary C. Croarkin, of the NBS Statistical Engineering Laboratory, for evaluating the function $F(z)$, utilizing the 7090 computer of the NBS Computation Laboratory; Mr. P. L. Rice for providing the data of section 3.1 ; Dr. J. S. Hunter, editor of "Technometrics" for the permission to reproduce table 1 ; and the Principia Press of Trinity University, San Antonio, Tex., for the permission to reproduce table 2 .

\section{References}

Davis, H. T. (1933), Tables of the Higher Mathematical Function, [1] (Principia Press, Bloomington).

Davis, H. T. (1935), Tables of the Higher Mathematical Functions, [2] (Principia Press, Bloomington).

Fisher, R. A. (1922), On the mathematical foundations of theoretical statistics, Phil. Trans. Roy. Soc. London, Ser. A222, 309-368. Reprinted in Fisher, R. A., Contributions to Mathematical Statistics (John Wiley \& Sons, Inc., New York, N.Y., 1950).

Greenwood, J. A., and D. Durand (1960), Aids for fitting the gamma distribution by maximum likelihood, Technometrics $2,55-65$.

Williamson, D. A., V. L. Fuller, A. G. Longley, and P. L. Rice (1960), A summary of VHF and UHF tropospheric transmission loss data and their long-term variability, NBS Tech. Note No. 43.

(Paper 67D6-304) 